\title{
THE PROPAGATION OF THERMAL STRESSES IN A SEMI-INFINITE MEDIUM
}

\author{
by F. J. LOCKETT \\ (Received 12th January 1960)
}

\section{Introduction}

When an elastic solid is subjected to a dynamical system of surface or body forces, not all of the work done by these forces is employed in deforming the material. The remainder is converted into heat energy producing a distribution of temperature throughout the body. Similarly the application of a surface temperature distribution, or the introduction of heat sources within the body, produces elastic as well as thermal effects. Thus we see that in the dynamical case there is a link between these two types of condition-thermal and elastic. The mathematical laws expressing this physical interaction take the form

$$
\begin{array}{r}
\nabla^{2} u+\left(\beta^{2}-1\right) \operatorname{grad} \Delta-b \operatorname{grad} \theta+X=a \ddot{u}, \\
\Theta+\nabla^{2} \theta=f \dot{\theta}+g \Delta, \ldots \ldots \ldots \ldots \ldots
\end{array}
$$

where $u$ and $\theta$ represent respectively the displacement vector and the temperature distribution, $\boldsymbol{X}$ and $\Theta$ are respectively the body forces and heat sources, $\nabla^{2}$ is the Laplacian operator, $\Delta=u_{i, i}$ and $\beta, b, a, f$ and $g$ are constants. These equations are due to Biot (1) and have been written in a particular dimensionless form due to Sneddon and Berry (6). Equation (1.2), which is a modified form of the classical heat conduction equation, was previously derived by Voigt (8) and by Jeffreys (4).

There are two special cases of the thermoelastic equations which have received considerable attention in the literature. The first is the steady-state problem for which the right-hand sides of equations (1.1) and (1.2) are taken to be zero, and the second is the classical problem in which the term $\partial \Delta / \partial t$ is omitted from equation (1.2), i.e. the classical heat conduction equation is used. Both of these special classes of problem are simpler than the one posed by the complete interlinked equations (1.1) and (1.2), since the special cases can be solved in two steps-the determination of $\theta$ from (1.2) followed by the evaluation of $u$ from (1.1). In the linked theory this is not possible, and equations (1.1) and (1.2) have to be solved simultaneously.

For the infinite medium, solutions to these equations. have been given by Eason and Sneddon (3) in the case of heat sources, and by Sneddon and Lockett (7) in the case of transient body forces. The results of these two papers may be summarised by saying that the displacement field $u=\left(u_{1}, u_{2}, u_{3}\right)$ 
and temperature distribution $\theta$ due to a combination of body forces $X=\left(X_{1}\right.$, $X_{2}, X_{3}$ ) and heat sources $\Theta$ are given by

$$
\begin{array}{r}
\bar{u}_{i}=\frac{\bar{X}_{i}}{\xi^{2}-a \omega^{2}}-\frac{\left\{\left(\beta^{2}-1\right)\left(\xi^{2}-i \omega f\right)-i \omega b g\right\} \xi_{i} \xi_{r} \bar{X}_{r}}{\left(\xi^{2}-a \omega^{2}\right) \mathscr{D}}+\frac{i b \xi_{i} \bar{\Theta}}{\mathscr{D}}, \\
\bar{\theta}=\frac{\omega g \xi_{r} \bar{X}_{r}+\left(\beta^{2} \xi^{2}-a \omega^{2}\right) \bar{\Theta}}{\mathscr{D}}, \ldots \ldots \ldots \ldots \ldots \ldots \ldots \ldots \ldots \ldots
\end{array}
$$

where

$$
\begin{array}{r}
\xi^{2}=\xi_{1}^{2}+\xi_{2}^{2}+\xi_{3}^{2}, \ldots \ldots \ldots \ldots \ldots \\
\mathscr{D}=\left(\beta^{2} \xi^{2}-a \omega^{2}\right)\left(\xi^{2}-i \omega f\right)-i \omega b g \xi^{2},
\end{array}
$$

and the bar denotes the four-dimensional complex Fourier transform

$$
\begin{aligned}
& \bar{F}\left(\xi_{1}, \xi_{2}, \xi_{3}, \omega\right) \\
& \quad=\frac{1}{4 \pi^{2}} \iiint \int_{-\infty}^{\infty} F\left(x_{1}, x_{2}, x_{3}, t\right) \exp \left\{i\left(\xi_{1} x_{1}+\xi_{2} x_{2}+\xi_{3} x_{3}+\omega t\right)\right\} d x_{1} d x_{2} d x_{3} d t .
\end{aligned}
$$

These results assume that all the unknowns and their derivatives vanish at infinity.

Using the system of units adopted in equations (1.1) and (1.2), the normal components of stress are given by

$$
\sigma_{i}=\left(\beta^{2}-2\right) \Delta-b \theta+2 \partial u_{i} / \partial x_{i}
$$

(no summation implied in the last term), and the shear components are

$$
\tau_{i j}=\partial u_{i} / \partial x_{j}+\partial u_{j} / \partial x_{i}
$$

\section{Statement of the Problem}

In this paper we shall consider the semi-infinite medium $x_{3} \geqq 0$ subjected to body forces $\boldsymbol{F}=\left(F_{1}, F_{2}, F_{3}\right)$ and a heat source distribution $\Phi$. Further there may be any set of physically possible ordinary boundary conditions on the boundary $x_{3}=0$. We shall therefore look for solutions to the linked equations (1.1) and (1.2) subject to the specification on $x_{3}=0$ of three of the quantities $u_{1}, u_{2}, u_{3}, \sigma_{3}, \tau_{31}, \tau_{32}$ plus one of the quantities $\theta, \partial \theta / \partial x_{3},\left(\partial \theta / \partial x_{3}+h \theta\right)$.

The standard method of using a four-dimensional integral transform on the given equations (a sine or cosine transform in the $x_{3}$-direction) will only work in certain special cases, because of our lack of knowledge of the behaviour of many of the unknowns and their derivatives at $x_{3}=0$. (For an example where this does work see Eason and Sneddon (3).) Further, the method of transforming the equations with respect to $x_{1}, x_{2}$ and $t$, and then solving the resulting four differential equations in the single variable $x_{3}$, is too cumbersome. Instead, we shall use the method described in the next section. 


\section{Method of Solution}

We wish to find expressions for $u$ and $\theta$ which, in the region $x_{3}>0$, satisfy the equations (1.1) and (1.2) with $X=F$ and $\Theta=\Phi$ and which satisfy the given boundary conditions on $x_{3}=0$. Instead we look for solutions which satisfy equations (1.1) and (1.2) in the whole space $-\infty \leqq x_{3} \leqq \infty$ when

$$
\begin{gathered}
X_{i}=F_{i}+(2 \pi)^{\frac{1}{2}} P_{i}\left(x_{1}, x_{2}, t\right) \delta\left(x_{3}\right) \\
\Theta=\Phi+(2 \pi)^{\frac{1}{2}} \Psi\left(x_{1}, x_{2}, t\right) \delta\left(x_{3}\right)
\end{gathered}
$$

where $\delta(x)$ is the Dirac Delta function. Thus we consider the infinite medium subjected to $\boldsymbol{F}$ and $\Phi$ in the region $x_{3}>0$ and to additional body forces $(2 \pi)^{ \pm} \boldsymbol{P}$ and heat sources $(2 \pi)^{ \pm} \Psi$ concentrated on the plane $x_{3}=0$. We shall then choose $\boldsymbol{P}$ and $\Psi$ in such a way that the boundary conditions of the original problem are satisfied on $x_{3}=0$.

Within the region $x_{3}>0$ the solutions to the modified problem satisfy the equations (1.1), (1.2) with $X=F$ and $\Theta=\Phi\left(\right.$ since $\delta\left(x_{3}\right)=0$ for $x_{3}>0$ ) and on the plane $x_{3}=0$ the given boundary conditions are satisfied. Thus for $x_{3}>0$ the solutions to the modified problem are the solutions to the original problem. The concentrated forces and sources $P$ and $\Psi$ could have been taken off the boundary $x_{3}=0$ by considering the Dirac Delta functions in the form $\delta\left(x_{3}+d\right)$ where $d>0$, so that they lie on the plane $x_{3}=-d$. However, when the analysis is carried through, we find that the solutions are independent of $d$, which we would expect on physical grounds, and so we content ourselves here with the case $d=0$.

The method described above is a particular case of a method due to Lockett (5) for solving a wider class of problems.

\section{Solution in Rectangular Cartesian Coordinates}

For the expressions (3.1) we find that

$$
\bar{X}_{i}=\bar{F}_{i}+P_{i}^{0}, \quad \bar{\Theta}=\bar{\Phi}+\Psi^{0},
$$

where $\vec{f}$ is defined by (1.7) and $f^{0}$ is the transform with respect to $x_{1}, x_{2}, t$ only. That is

$$
f^{0}=\frac{1}{(2 \pi)^{\frac{3}{2}}} \iiint_{-\infty}^{\infty} f \exp \left\{i\left(\xi_{1} x_{1}+\xi_{2} x_{2}+\omega t\right)\right\} d x_{1} d x_{2} d t
$$

It is obvious from (1.7) and (4.2) that

so that

$$
\bar{f}=\frac{1}{(2 \pi)^{\frac{1}{2}}} \int_{-\infty}^{\infty} f^{0} \exp \left(i \xi_{3} x_{3}\right) d x_{3}
$$

$$
f^{0}=\frac{1}{(2 \pi)^{\frac{1}{2}}} \int_{-\infty}^{\infty} f \exp \left(-i \xi_{3} x_{3}\right) d \xi_{3} \text {. }
$$


Using the expressions (1.3), (1.4) and (4.1) we can write the solutions to the modified problem in the form

where

$$
u_{i}=u_{i 1}+u_{i 2}, \quad \theta=\theta_{1}+\theta_{2} \text {, }
$$

$$
\begin{aligned}
\bar{u}_{i 1} & =\frac{\bar{F}_{i}}{\xi^{2}-a \omega^{2}}-\frac{\left\{\left(\beta^{2}-1\right)\left(\xi^{2}-i \omega f\right)-i \omega b g\right\} \xi_{i} \xi_{q} \bar{F}_{q}}{\left(\xi^{2}-a \omega^{2}\right) \mathscr{D}}+\frac{i b \xi_{i} \Phi}{\mathscr{D}} \\
\bar{\theta}_{1} & =\frac{\omega g \xi_{q} \bar{F}_{q}+\left(\beta^{2} \xi^{2}-a \omega^{2}\right) \bar{\Phi}}{\mathscr{D}}
\end{aligned}
$$

and

$$
\begin{aligned}
\bar{u}_{i 2} & =\frac{P_{i}^{0}}{\xi^{2}-a \omega^{2}}-\frac{\left\{\left(\beta^{2}-1\right)\left(\xi^{2}-i \omega f\right)-i \omega b g\right\} \xi_{i} \xi_{q} P_{q}^{0}}{\left(\xi^{2}-a \omega^{2}\right) \mathscr{D}}+\frac{i b \xi_{i} \Psi^{0}}{\mathscr{D}} \\
\bar{\theta}_{2} & =\frac{\omega g \xi_{q} P_{q}^{0}+\left(\beta^{2} \xi^{2}-a \omega^{2}\right) \Psi^{0}}{\mathscr{D}}
\end{aligned}
$$

Since in any particular problem $F_{i}$ and $\Phi$ are known, $\bar{u}_{i 1}$ and $\bar{\theta}_{1}$ can be evaluated and can therefore be treated as known functions. From (4.3) and (4.6)

$$
\begin{aligned}
& (2 \pi)^{\frac{1}{2}} u_{i 2}^{0} \\
& =P_{i}^{0} \int_{-\infty}^{\infty} \frac{e^{-i \xi_{3} x_{3}}}{\xi^{2}-a \omega^{2}} d \xi_{3}-P_{q}^{0} \int_{-\infty}^{\infty} \frac{\left\{\left(\beta^{2}-1\right)\left(\xi^{2}-i \omega f\right)-i \omega b g\right\} \xi_{i} \xi_{q}}{\left(\xi^{2}-a \omega^{2}\right) \mathscr{D}} e^{-i \xi_{3} x_{3}} d \xi_{3} \\
& +i b \Psi^{0} \int_{-\infty}^{\infty} \frac{\xi_{i} e^{-i \xi_{3} x_{3}}}{\mathscr{D}} d \xi_{3}
\end{aligned}
$$

$$
(2 \pi)^{\frac{1}{2}} \theta_{2}^{0}=\omega g P_{q}^{0} \int_{-\infty}^{\infty} \frac{\xi_{q} e^{-i \xi_{3} x_{3}}}{\mathscr{D}} d \xi_{3}+\Psi^{0} \int_{\dot{-}_{-\infty}}^{\infty} \frac{\left(\beta^{2} \xi^{2}-a \omega^{2}\right)}{\mathscr{D}} e^{-i \xi_{3} x_{3}} d \xi_{3},
$$

since $P_{i}^{0}$ and $\Psi^{0}$ are not functions of $\xi_{3}$. If we now write

$$
\xi^{2}-a \omega^{2}=\xi_{3}^{2}+k_{1}^{2}
$$

so that

$$
k_{1}^{2}=\eta^{2}-a \omega^{2} \text { where } \eta^{2}=\xi_{1}^{2}+\xi_{2}^{2}
$$

and put

$$
\mathscr{D}=\beta^{2}\left(\xi_{3}^{2}+k_{2}^{2}\right)\left(\xi_{3}^{2}+k_{3}^{2}\right),
$$

the integrals in (4.7) can be evaluated by reducing the integrands by partial fractions. If we evaluate the integrals for $x_{3}>0$ (the only range in which we are interested) and, for convenience, introduce new unknowns $A, B, C$ and $D$ which depend on $P_{i}^{0}$ and $\Psi^{0}$, then we find that

$$
\begin{aligned}
u_{12}^{0} & =i k_{1} A e^{-k_{1} x_{3}}+i b \xi_{1} C e^{-k_{2} x_{3}}+i b \xi_{1} D e^{-k_{3} x_{3}}, \\
u_{22}^{0} & =i k_{1} B e^{-k_{1} x_{3}}+i b \xi_{2} C e^{-k_{2} x_{3}}+i b \xi_{2} D e^{-k_{3} x_{3}}, \\
u_{32}^{0} & =\left(\xi_{1} A+\xi_{2} B\right) e^{-k_{1} x_{3}}+b k_{2} C e^{-k_{2} x_{3}}+b k_{3} D e^{-k_{3} x_{3}}, \\
\theta_{2}^{0} & =\left(\beta^{2} \eta^{2}-a \omega^{2}-\beta^{2} k_{2}^{2}\right) C e^{-k_{2} x_{3}}+\left(\beta^{2} \eta^{2}-a \omega^{2}-\beta^{2} k_{3}^{2}\right) D e^{-k_{3} x_{3}} .
\end{aligned}
$$


Thus, using (4.4), the three-dimensional transforms of the displacement field and the temperature distribution are given by

$$
\begin{aligned}
& u_{1}^{0}=u_{11}^{0}+i k_{1} A e^{-k_{1} x_{3}}+i b \xi_{1} C e^{-k_{2} x_{3}}+i b \xi_{1} D e^{-k_{3} x_{3}}, \\
& u_{2}^{0}=u_{21}^{0}+i k_{1} B e^{-k_{1} x_{3}}+i b \xi_{2} C e^{-k_{2} x_{3}}+i b \xi_{2} D e^{-k_{3} x_{3}}, \\
& u_{3}^{0}=u_{31}^{0}+\left(\xi_{1} A+\xi_{2} B\right) e^{-k_{1} x_{3}}+b k_{2} C e^{-k_{2} x_{3}}+b k_{3} D e^{-k_{3} x_{3}}, \\
& \theta^{0}=\theta_{1}^{0}+\left(\beta^{2} \eta^{2}-a \omega^{2}-\beta^{2} k_{2}^{2}\right) C e^{-k_{2} x_{3}}+\left(\beta^{2} \eta^{2}-a \omega^{2}-\beta^{2} k_{3}^{2}\right) D e^{-k_{3} x_{3}},
\end{aligned}
$$

where $u_{i 1}^{0}$ and $\theta_{i}^{0}$ can be found using (4.3) and (4.5).

In any particular problem we have only now to apply (4.12) to the transformed boundary conditions to obtain four equations giving the values of $A$, $B, C$ and $D$. Substituting these values back into (4.12) and applying the transform inverse to (4.2) we obtain expressions for the required quantities $u$ and $\theta$.

Transforms of stress components. Since the boundary conditions to many problems specify one or more of the components of stress $\sigma_{3}, \tau_{31}, \tau_{32}$ we give here their three-dimensional transforms:

$$
\begin{aligned}
\sigma_{3}^{0} & =-i\left(\beta^{2}-2\right)\left(\xi_{1} u_{1}^{0}+\xi_{2} u_{2}^{0}\right)-b \theta^{0}+\beta^{2} \partial u_{3}^{0} / \partial x_{3}, \\
\tau_{31}^{0} & =\partial u_{1}^{0} / \partial x_{3}-i \xi_{1} u_{3}^{0}, \\
\tau_{32}^{0} & =\partial u_{2}^{0} / \partial x_{3}-i \xi_{2} u_{3}^{0} .
\end{aligned}
$$

\section{Example}

As an example of the use of the equations derived above we shall consider the problem considered by Eason and Sneddon (3). Here $\boldsymbol{F}=\mathbf{0}$ and the boundary conditions are $u_{1}=u_{2}=\sigma_{3}=0, \theta=\theta_{0}\left(x_{1}, x_{2}, t\right)$ on $x_{3}=0$. A heat source $\Phi\left(x_{1}, x_{2}, x_{3}, t\right)$ is assumed to be acting in the medium $x_{3}>0$.

Thus from (4.5)

$$
\bar{u}_{i 1}=i b \xi_{i} \bar{\Phi} / \mathscr{D}, \quad \bar{\theta}_{1}=\left(\beta^{2} \xi^{2}-a \omega^{2}\right) / \bar{\Phi} \mathscr{D}
$$

and the boundary conditions can be written

$$
\begin{aligned}
& \frac{i b \xi_{1}}{(2 \pi)^{\frac{1}{2}}} \int_{-\infty}^{\infty} \frac{\Phi}{\mathscr{D}} d \xi_{3}+i k_{1} A+i b \xi_{1} C+i b \xi_{1} D=0, \\
& \frac{i b \xi_{2}}{(2 \pi)^{\frac{1}{2}}} \int_{-\infty}^{\infty} \frac{\bar{\Phi}}{\mathscr{D}} d \xi_{3}+i k_{1} B+i b \xi_{2} C+i b \xi_{2} D=0, \\
& \begin{aligned}
\frac{-b\left(2 \eta^{2}-a \omega^{2}\right)}{(2 \pi)^{\frac{1}{2}}} \int_{-\infty}^{\infty} \frac{\bar{\Phi}}{\mathscr{D}} d \xi_{3}-2 k_{1} \xi_{1} A-2 k_{1} \xi_{2} B-b\left(2 \eta^{2}-a \omega^{2}\right)(C+D)=0 \\
\frac{1}{(2 \pi)^{\frac{1}{2}}} \int_{-\infty}^{\infty} \frac{\left(\beta^{2} \xi^{2}-a \omega^{2}\right) \bar{\Phi}}{\mathscr{D}} d \xi_{3}+\left(\beta^{2} \eta^{2}-a \omega^{2}-\beta^{2} k_{2}^{2}\right) C \\
+\left(\beta^{2} \eta^{2}-a \omega^{2}-\beta^{2} k_{3}^{2}\right) D=\theta_{0}^{0}
\end{aligned}
\end{aligned}
$$


from which we find that $A=B=0$ and

$$
\begin{aligned}
& C=\left(k_{2}^{2}-k_{3}^{2}\right)^{-1}\left\{-\beta^{-2} \theta_{0}^{0}+(2 \pi)^{-\frac{1}{2}} \int_{-\infty}^{\infty} \frac{\left(\xi_{3}^{2}+k_{3}^{2}\right) \Phi}{\mathscr{D}} d \xi_{3}\right\}, \\
& D=\left(k_{2}^{2}-k_{3}^{2}\right)^{-1}\left\{\beta^{-2} \theta_{0}^{0}-(2 \pi)^{-\frac{1}{2}} \int_{-\infty}^{\infty} \frac{\left(\xi_{3}^{2}+k_{2}^{2}\right) \Phi}{\mathscr{D}} d \xi_{3}\right\} .
\end{aligned}
$$

We may now use (4.12) and the inverse transforms to show that

where

$$
\boldsymbol{u}=-\operatorname{grad} \psi,
$$

$$
\begin{aligned}
\psi=\frac{b}{4 \pi^{2}} \iiint_{-\infty}^{\infty} e^{-i\left(\xi_{1} x_{1}+\xi_{2} x_{2}+\omega t\right)} d \xi_{1} d \xi_{2} d \omega & \left\{\int_{-\infty}^{\infty} e^{-i \xi_{3} x_{3}} \frac{\Phi}{\mathscr{D}} d \xi_{3}\right. \\
& \left.\left.+(2 \pi)^{\frac{1}{2}\left(C e^{-k_{2} x_{3}}\right.}+D e^{-k_{3} x_{3}}\right)\right\} .
\end{aligned}
$$

This is a formal solution to the problem, and it can be shown that it is equivalent to the result obtained by Eason and Sneddon. However, it should be noted that the present method will produce formal solutions to any of the problems mentioned earlier.

\section{Solutions for the Axially Symmetrical Problem}

The solutions to this problem could be found by running through a similar procedure to the one outlined in $\S 4$, using the forms of the equations when expressed in cylindrical polar coordinates. However, it is not a difficult matter to obtain them directly from the results expressed in rectangular coordinates.

We denote by $u_{\mathrm{r}}$ and $w$ the components of displacement in the $r$ and $z$ directions, and use $\psi$ to denote the polar coordinate. The components of the body force are denoted by $F_{r}$ and $F_{z}$ and the temperature distribution and heat sources are represented by $\theta$ and $\Phi$ as before.

We define the transforms

and

$$
\begin{aligned}
\left(u_{r}^{0}, F_{r}^{0}\right) & =\frac{1}{(2 \pi)^{\frac{1}{2}}} \int_{-\infty}^{\infty} e^{i \omega t} d t \int_{0}^{\infty} r\left(u_{r}, F_{r}\right) J_{1}(\xi r) d r, \ldots \ldots \\
\left(w^{0}, \theta^{0}, F_{z}^{0}, \Phi^{0}\right) & =\frac{1}{(2 \pi)^{\frac{1}{2}}} \int_{-\infty}^{\infty} e^{i \omega t} d t \int_{0}^{\infty} r\left(w, \theta, F_{z}, \Phi\right) J_{0}(\xi r) d r
\end{aligned}
$$

$$
f=\frac{1}{(2 \pi)^{\frac{1}{2}}} \int_{-\infty}^{\infty} f^{0} e^{i \zeta z} d z
$$

If we make the following substitutions from the rectangular system to the polar system

$$
\left.\begin{array}{lll}
x_{1}=r \cos \psi, & x_{2}=r \sin \psi, & x_{3}=z \\
u_{1}=u_{r} \cos \psi, & u_{2}=u_{r} \sin \psi, & u_{3}=w \\
F_{1}=F_{r} \cos \psi, & F_{2}=F_{r} \sin \psi, & F_{3}=F_{z}
\end{array}\right\}
$$


and put

$$
\xi_{1}=\xi \cos \phi, \quad \xi_{2}=\xi \sin \phi, \quad \xi_{3}=\zeta
$$

then we find that, for instance

$$
\begin{aligned}
& \xi_{1} \bar{F}_{1}+\xi_{2} \bar{F}_{2} \\
& =\frac{1}{(2 \pi)^{2}} \iiint \int_{-\infty}^{\infty}\left(\xi_{1} F_{1}+\xi_{2} F_{2}\right) \exp \left\{i\left(\xi_{1} x_{1}+\xi_{2} x_{2}+\xi_{3} x_{3}+\omega t\right)\right\} d x_{1} d x_{2} d x_{3} d t \\
& =\frac{1}{(2 \pi)^{2}} \iiint \int_{-\infty}^{\infty} \xi F_{r} \cos (\phi-\psi) \exp \left\{i\left(\xi_{1} x_{1}+\xi_{2} x_{2}+\xi_{3} x_{3}+\omega t\right)\right\} d x_{1} d x_{2} d x_{3} d t \\
& \quad=\frac{1}{2 \pi} \iint_{-\infty}^{\infty} e^{i(\zeta z+\omega t)} d z d t \int_{0}^{\infty} \xi F_{r} r d r \frac{1}{2 \pi} \int_{0}^{2 \pi} \cos (\phi-\psi) e^{i \xi r \cos (\phi-\psi)} d \psi \\
& =\frac{i \xi}{2 \pi} \iint_{-\infty}^{\infty} e^{i(\zeta z+\omega t)} d z d t \int_{0}^{\infty} r F_{r} J_{1}\left(\xi_{r}\right) d r .
\end{aligned}
$$

Therefore

Similarly

$$
\bar{F}_{r}=\left(\xi_{1} \bar{F}_{1}+\xi_{2} \bar{F}_{2}\right) / i \xi
$$

$$
\bar{u}_{r}=\left(\xi_{1} \bar{u}_{1}+\xi_{2} \bar{u}_{2}\right) / i \xi, \quad \bar{w}=\bar{u}_{3}, \quad \bar{F}_{z}=\bar{F}_{3},
$$

and $\bar{\theta}$ and $\bar{\Phi}$ defined by (6.2) and (6.3) are identical to the definitions given by (1.7). A similar set of relations holds where $\bar{f}$ is replaced by $f^{\circ}$. From these expressions and equations (4.5) and (4.12) it is easily seen that $u_{r}, w$ and $\theta$ are given by

$$
\left.\begin{array}{l}
u_{r}^{0}=u_{r 1}^{0}+k_{1} L e^{-k_{1} z}+b \xi M e^{-k_{2} z}+b \xi N e^{-k_{3} z} \\
w^{0}=w_{1}^{0}+\xi L e^{-k_{1} z}+b k_{2} M e^{-k_{2} z}+b k_{3} N e^{-k_{3} z} \\
\theta^{0}=\theta_{1}^{0}+\left(\beta^{2} \xi^{2}-a \omega^{2}-\beta^{2} k_{2}^{2}\right) M e^{-k_{2} z}+\left(\beta^{2} \xi^{2}-a \omega^{2}-\beta^{2} k_{3}^{2}\right) N e^{-k_{3} z}
\end{array}\right\}
$$

where

$$
\left.\begin{array}{rl}
k_{1}^{2} & =\xi^{2}-a \omega^{2} \\
\beta^{2}\left(\zeta^{2}+k_{2}^{2}\right)\left(\zeta^{2}+k_{3}^{2}\right) & =\left(\beta^{2} \xi^{2}+\beta^{2} \zeta^{2}-a \omega^{2}\right)\left(\xi^{2}+\zeta^{2}-i \omega f\right)-i \omega b g\left(\xi^{2}+\zeta^{2}\right)
\end{array}\right\}
$$

and $u_{r 1}^{0}, w^{0}, \theta_{1}^{0}$ are obtained from (4.3) and the expressions

$$
\begin{aligned}
\bar{u}_{r 1} & =\frac{\bar{F}_{r}}{\left(\xi^{2}+\zeta^{2}-a \omega^{2}\right)}+\frac{i \xi\left\{\left(\beta^{2}-1\right)\left(\xi^{2}+\zeta^{2}-i \omega f\right)-i \omega b g\right\}\left(i \xi \bar{F}_{r}+\zeta \bar{F}_{z}\right)}{\left(\xi^{2}+\zeta^{2}-a \omega^{2}\right) \mathscr{D}}+\frac{b \xi \bar{\Phi}}{\mathscr{D}} \\
\bar{w}_{1} & =\frac{\bar{F}_{z}}{\left(\xi^{2}+\zeta^{2}-a \omega^{2}\right)}-\frac{\left\{\left(\beta^{2}-1\right)\left(\xi^{2}+\zeta^{2}-i \omega f\right)-i \omega b g\right\}\left(i \xi \bar{F}_{r}+\zeta \bar{F}_{z}\right)}{\left(\xi^{2}+\zeta^{2}-a \omega^{2}\right) \mathscr{D}}+\frac{i b \zeta \bar{\Phi}}{\mathscr{D}} \\
\bar{\theta}_{1} & =\frac{i \omega g \xi \bar{F}_{r}+\omega g \zeta \bar{F}_{z}+\left(\beta^{2} \xi^{2}+\beta^{2} \zeta^{2}-a \omega^{2}\right) \Phi}{\mathscr{D}}
\end{aligned}
$$


The final results to any particular problem can now be found by

(i) applying the transformed boundary conditions to equations (6.6) to obtain $L, M$ and $N$.

(ii) calculating $u_{r 1}^{0}, w_{1}^{0}, \theta_{1}^{0}$ from (4.3) and (6.8)

(iii) substituting these values into (6.6)

(iv) inverting (6.6) using the transforms inverse to (6.1) and (6.2).

Transforms of the stress components. With the notation used above the two-dimensional transforms of the components of stress $\sigma_{z}$ and $\tau_{r z}$ are

$$
\begin{aligned}
& \sigma_{z}^{0}=\frac{1}{(2 \pi)^{\frac{1}{2}}} \int_{-\infty}^{\infty} e^{i \omega t} d t \int_{0}^{\infty} r \sigma_{z} J_{0}(\xi r) d r=\left(\beta^{2}-2\right) \xi u_{r}^{0}-b \theta^{0}+\beta^{2} \partial w^{0} / \partial z, \\
& \tau_{r z}^{0}=\frac{1}{(2 \pi)^{\frac{1}{t}}} \int_{-\infty}^{\infty} e^{i \omega t} d t \int_{0}^{\infty} r \tau_{r z} J_{1}(\xi r) d r=\partial u_{r}^{0} / \partial z-\xi w^{0} .
\end{aligned}
$$

\section{Simple Numerical Example}

With the equations derived above it is possible to write down a formal solution to any of the problems mentioned in $\S 2$. However, in most problems, it will be necessary to use numerical methods to put the results in a meaningful form. We shall be satisfied here to look at the result of taking into account the coupling effects for a problem which, though not of obvious practical interest, reduces the numerical work to a minimum.

Consider a semi-infinite elastic medium resting upon a rigid frictionless foundation, so that $w=\tau_{r z}=0$ on $z=0$. We suppose too that the rate of change of temperature across the boundary is known, i.e. $\partial \theta / \partial z=\phi(r, t)$ on $z=0$. We wish to find the pressure exerted by the solid onto the foundation. (It is assumed that the medium is prevented from separating from the foundation.) The equations for the evaluation of $L, M$ and $N$ take the form

so that

$$
\begin{aligned}
& \xi L+b k_{2} M+b k_{3} N=0, \\
& \left(\xi^{2}+k_{1}^{2}\right) L+2 b \xi k_{2} M+2 b \xi k_{3} N=0, \\
& -\left(\beta^{2} \xi^{2}-a \omega^{2}-\beta^{2} k_{2}^{2}\right) k_{2} M-\left(\beta^{2} \xi^{2}-a \omega^{2}-\beta^{2} k_{3}^{2}\right) k_{3} N=\phi^{0},
\end{aligned}
$$

$$
L=0, \quad k_{2} M=-k_{3} N=\phi^{0} / \beta^{2}\left(k_{2}^{2}-k_{3}^{2}\right) .
$$

We now choose the problem in which $\phi$ is equal to

$$
P B e^{A t} J_{0}(B r)
$$

(where $P, A, B$ are constants), which is obtained by placing

$$
\phi^{0}=(2 \pi)^{\frac{1}{2}} P \delta(\omega-i A) \delta(\xi-B)
$$

in the formula for $\phi$ in terms of its transform. Using (6.6), (7.1) and (7.2) we find the solutions

$$
\begin{aligned}
u_{r}= & P B^{2} b\left\{\beta^{2}\left(k_{2}^{2}-k_{3}^{2}\right) k_{2} k_{3}\right\}^{-1}\left(k_{3} e^{-k_{22} z}-k_{2} e^{-k_{3} z}\right) e^{A t} J_{1}(B r), \\
w= & P B b\left\{\beta^{2}\left(k_{2}^{2}-k_{3}^{2}\right)\right\}^{-1}\left(e^{-k_{2} z}-e^{-k_{3} z}\right) e^{A t} J_{0}(B r), \ldots \ldots \ldots \ldots \ldots \ldots \ldots \ldots \ldots \ldots \ldots \ldots \ldots \ldots \ldots \ldots \ldots \ldots \ldots \\
\theta= & P B\left\{\beta^{2}\left(k_{2}^{2}-k_{3}^{2}\right)\right\}^{-1} \\
& \left\{k_{2}^{-1}\left(\beta^{2} B^{2}-a A^{2}-\beta^{2} k_{2}^{2}\right) e^{-k_{2} z}-k_{3}^{-1}\left(\beta^{2} B^{2}-a A^{2}-\beta^{2} k_{3}^{2}\right) e^{-k_{3} z}\right\} e^{A t} J_{0}(B r),
\end{aligned}
$$


where $k_{2}$ and $k_{3}$, are to be evaluated for $\omega=i A, \xi=B$. This is not a rigorous derivation of these results, but it can be verified that they satisfy all the required equations and conditions (remembering that $k_{2}$ and $k_{3}$ satisfy (6.7)).

Using (6.9) it is also easily seen that on the boundary $z=0$

$$
\sigma_{z}=\frac{P B b\left(2 B^{2}+a A^{2}\right)}{\beta^{2}\left(k_{2}+k_{3}\right) k_{2} k_{3}} e^{A t} J_{0}(B r) .
$$

Thus, in this solution, the discrepancy between the result obtained using the linked equations and that obtained using the uncoupled equations $(g=0)$ occurs only in the constant multiplying factor, and the ratio of these constants is

$$
R=\left\{\frac{\left\{\left(k_{2}+k_{3}\right) k_{2} k_{3}\right\}_{g=0}}{\left(k_{2}+k_{3}\right) k_{2} k_{3}}\right\}_{\substack{\omega=i A \\ \xi=B}} .
$$

It is more convenient to use now the system of units due to Chadwick and Sneddon (2), which corresponds to placing $a=\beta^{2}, f=1, \varepsilon=b g / \beta^{2}$. In this system the units of length and time are very small $\left(\sim 10^{-8}\right)$. Thus in formulating a problem which may be encountered in practice, we need to take $A, B \ll 1$. Otherwise the variations of the physical quantities with time would be too rapid, and the $r$-dependence would be too localised.

The ratio can now be written as

$$
R=\frac{\left\{\left(k_{2}+k_{3}\right) k_{2} k_{3}\right\}_{\varepsilon}=0}{\left(k_{2}+k_{3}\right) k_{2} k_{3}}
$$

where $k_{2}$ and $k_{3}$ are given by

$$
\left(\zeta^{2}+k_{2}^{2}\right)\left(\zeta^{2}+k_{3}^{2}\right)=\left(\zeta^{2}+B^{2}+A^{2}\right)\left(\zeta^{2}+B^{2}+A\right)+A \varepsilon\left(\zeta^{2}+B^{2}\right)
$$

The following table shows, for a range of values of $A$ and $B$, and with $\varepsilon=0.05$ (the value for copper is 0.017 and the value for lead is 0.073 ), the percentage

\begin{tabular}{|c|c|c|c|c|c|c|}
\hline$A$ & $10^{-5}$ & $10^{-10}$ & $10^{-15}$ & $10^{-20}$ & $10^{-25}$ & $10^{-30}$ \\
\hline $\begin{array}{l}10^{-5} \\
10^{-10} \\
10^{-15} \\
10^{-20} \\
10^{-25} \\
10^{-30}\end{array}$ & $\begin{array}{l}3 \cdot 6 \\
2 \cdot 4 \\
2 \cdot 4 \\
2 \cdot 4 \\
2 \cdot 4 \\
2 \cdot 4\end{array}$ & $\begin{array}{l}1 \cdot 9 \\
3 \cdot 6 \\
2 \cdot 4 \\
2 \cdot 4 \\
2 \cdot 4 \\
2 \cdot 4\end{array}$ & $\begin{array}{l}0 \\
4 \cdot 8 \\
3 \cdot 6 \\
2 \cdot 4 \\
2 \cdot 4 \\
2 \cdot 4\end{array}$ & $\begin{array}{l}0 \\
1 \cdot 9 \\
4 \cdot 8 \\
3 \cdot 6 \\
2 \cdot 4 \\
2 \cdot 4\end{array}$ & $\begin{array}{l}0 \\
0 \\
4 \cdot 8 \\
4 \cdot 8 \\
3 \cdot 6 \\
2 \cdot 4\end{array}$ & $\begin{array}{l}0 \\
0 \\
1.9 \\
4.8 \\
4.8 \\
3.6\end{array}$ \\
\hline
\end{tabular}
error of the uncoupled solution with respect to the solution obtained from the linked equations (error $=100(1-R)$ ).

Percentage errors in the surface value of $\sigma_{z}$ 
From this table it is obvious that, as long as $A, B \ll 1$, the error depends only on a quantity $c$ defined by the relation $B^{c}=A$. It is easily seen that the maximum error is approximately 4.8 per cent. and occurs through the range $1<c<2$. Further for $c>2$ the error is negligible and it is approximately 2.4 per cent. for $c<1$ (but $c$ must not be so small as to violate the condition $A \ll 1)$.

\section{Acknowledgments}

I am indebted to Professor I. N. Sneddon for suggesting this problem.

The work was performed partly in The University of Glasgow with the aid of a Research Studentship awarded by the Department of Scientific and Industrial Research, and partly at Duke University, North Carolina, under Contract Number AF 18(600)-1341 with the Air Force Office of Scientific Research.

\section{REFERENCES}

(1) M. A. Biot, Thermoelasticity and irreversible thermodynamics, J. Appl. Phys., 27 (1956), 3, 240.

(2) P. Chadwick and I. N. Sneddon, Plane waves in an elastic solid conducting heat, J. Mech. Phys. Solids, 6 (1958), 223.

(3) G. EAsON and I. N. SNEDDon, The dynamic stresses produced in elastic bodies by uneven heating, Proc. Roy. Soc. Edin., 65 (1959), 143.

(4) H. Jefrreys, The thermodynamics of an elastic solid, Proc. Camb. Phil. Soc., 26 (1930), 101.

(5) F. J. LOCKETT, A method of deriving solutions of a class of boundary value problems, Proc. Edin. Math. Soc., 11 (1959), 147.

(6) I. N. Sneddon and D. S. Berry, The classical theory of elasticity, Hand. der Phys., Bd. 6, Berlin: Springer-Verlag (1958).

(7) I. N. Sneddon and F. J. Lockett, Propagation of thermal stresses in an infinite medium, Proc. Edin. Math. Soc., 11 (1959), 237.

(8) W. VoIgt, Lehrbuch der Kristallphysik, Berlin: Teubner-Verlag (1910).

\section{Department of Mathematics \\ UNIVERSITY OF GLASGOW}

Now at:

ARmament Research and Development Establishment, Fort Halstead, Sevenoaks, Kent. 